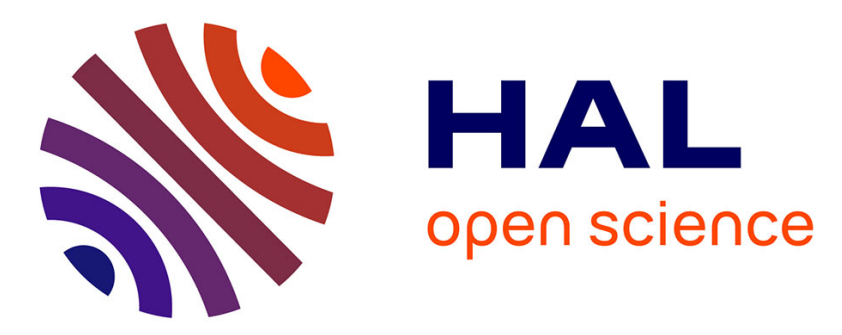

\title{
Measurement of Shock Wave and Particle Velocities in Shocked Dielectric Material from Millimeter-Wave Remote Sensing
}

Benoit Rougier, Hervé Aubert, Alexandre Lefrançois

\section{- To cite this version:}

Benoit Rougier, Hervé Aubert, Alexandre Lefrançois. Measurement of Shock Wave and Particle Velocities in Shocked Dielectric Material from Millimeter-Wave Remote Sensing. European Microwave Week, Sep 2018, Madrid, Spain. 9p. hal-01876708

\author{
HAL Id: hal-01876708 \\ https://hal.laas.fr/hal-01876708
}

Submitted on 18 Sep 2018

HAL is a multi-disciplinary open access archive for the deposit and dissemination of scientific research documents, whether they are published or not. The documents may come from teaching and research institutions in France or abroad, or from public or private research centers.
L'archive ouverte pluridisciplinaire HAL, est destinée au dépôt et à la diffusion de documents scientifiques de niveau recherche, publiés ou non, émanant des établissements d'enseignement et de recherche français ou étrangers, des laboratoires publics ou privés. 


\title{
Measurement of Shock Wave and Particle Velocities in Shocked Dielectric Material from Millimeter-Wave Remote Sensing
}

\author{
B. Rougier ${ }^{1,2}$, H. Aubert ${ }^{2}$, A. Lefrançois ${ }^{1}$ \\ ${ }^{1}$ CEA Gramat, France \\ ${ }^{2}$ LAAS CNRS, INPT, Toulouse, France
}

\begin{abstract}
A millimeter-wave remote sensing technique is used here as a noninvasive and continuous approach for the real-time measurement of shock wave velocity as well as the velocity of the shocked dielectric material during an impact. Experimental results obtained from planar symmetric impacts on PolyMethyl MethAcrylate (PMMA) cylinders are discussed and demonstrate that the proposed millimeter-wave remote sensing technique is highly convenient for deriving both the velocity of the shock wave and velocity of the shocked PMMA material. The proposed approach is applicable to any dielectric material subject to an impact and is an excellent candidate for deriving the equation of state of shocked materials.
\end{abstract}

\section{Introduction}

During an high speed mechanical impact on a dielectric material, two velocities are representative of the mechanical behavior of the material: the velocity of the shock wave and the particle velocity, that is, the velocity of the material behind the shock wave [1]. The measure of these quantities allows the determination of behavior laws for shocked materials for simulation purposes. Fiber Bragg Gratings [2] and shorting pins [3] are commonly used to measure the shock wave velocity, but such measurements are invasive. The particle velocity after an impact is classically derived from laser interferometry techniques [4], but the shocked material must be optically transparent. The simultaneous measurement of the shock wave and particle velocities can be achieved from X radiography [5], impedance matching [6] or electromagnetic particle velocity gauges [7]. However, these techniques can probe only optically transparent materials, may be invasive and/or require the knowledge of the dynamic properties of shocked materials (see, e.g., [1]). Since the early 1950s, radio-interferometry techniques have been applied to remotely investigate the shock wave velocity in dielectric materials subject to an impact [8]. They consist of probing shocked materials with a radio-frequency electromagnetic (EM) wave. The shock wave in such material acts as a semi-transparent dielectric interface and causes the reflection of the incident EM wave. From the analysis of the Doppler effect on the reflected wave, the shock wavefront velocity can be derived. This remote sensing technique is non-invasive and may be advantageously used for investigating physical or chemical phenomena in shocked materials during, for example, the shock-to-detonation transition [9]. Such remote derivation of the shock wave velocity is actually a well-known technique, but we show here that it is possible to extend the technique for the simultaneous derivation of both the particle and shock wave velocities without assumptions on the shocked materials permittivity. Indeed, as the shock wave creates a moving semi-transparent dielectric interface in the materials, a part of the incident EM wave is transmitted through this interface. This transmission allows sensing the shocked material behind the shock wave and consequently, may be used for measuring the particle velocity from Doppler effect on the reflected wave. Using such EM sensing approach, first attempts for deriving both the particle and 
Figure 1: Photo of the experimental setup (detail) showing the PMMA cylindrical sample under test, the conical Teflon applicator and the rectangular Teflon waveguide $(1.2 \mathrm{~mm} \times 2.4 \mathrm{~mm})$. The applicator is used here to inject into the PMMA sample a continuous millimeter-wave $(94 \mathrm{GHz})$.

shock wave velocities have been reported in [10]-[12], but they required assumptions on the permittivity of the shocked material. In this paper, the proposed millimeter-wave sensing technique does not require the prior knowledge of the shocked materials permittivity for estimating the particle and shock wave velocities in these materials. Experimental results obtained from plane symmetric impacts on PolyMethyl MethAcrylate (PMMA) cylinders are given for demonstrating that the proposed technique is highly convenient for simultaneously deriving the two velocities in the shocked PMMA material.

\section{Experimental setup and measurement results}

The experimental setup consists of a PolyMethyl MethAcrylate (PMMA) target with an aluminum coating at one side and a Teflon applicator at the center of the other side. The aluminum coating is used here to ensure the total reflection of the EM wave transmitted through the shock wavefront. The target is a PMMA dielectric cylinder of $30 \mathrm{~mm}$ in diameter and $15 \mathrm{~mm}$ in thickness. The applicator is a dielectric (Teflon) cone of $80 \mathrm{~mm}$ in length and $16 \mathrm{~mm}$ in diameter at its base. This cone is in contact with a dielectric (Teflon) waveguide $(1.2 \mathrm{~mm} \times 2.4 \mathrm{~mm})$ of $5 \mathrm{~m}$ in length. The waveguide is connected to a millimeter-wave interferometer operating at $94 \mathrm{GHz}$. The setup is placed at the muzzle of a light gas gun. At a time $t_{0}$, a PMMA impactor $(30 \mathrm{~mm}$ of diameter and $15 \mathrm{~mm}$ of thickness) is propelled by using the expansion of a pressurized tank of helium towards the dielectric material under test, with an impact velocity denoted by $V_{i m p}$. To the impact, a shock wave is generated in the dielectric material. The physics of dielectric materials under shock loading is out of the scope of this paper and is detailed, e.g., in [1]. The main phenomenon of interest here is the change in mass density occurring when the generated shock wave propagates along the material. This changes the permittivity and as a result, creates a moving dielectric interface inside the material. When an electromagnetic wave (generated here by millimeter-wave source) 


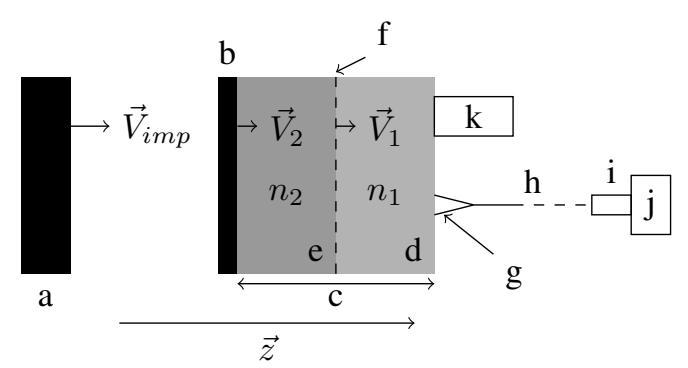

Figure 2: Sketch of the experimental setup configuration- $a$ : Impactor, $b$ : Aluminum coating, $c$ : Target, $d$ : Unshocked material, $e$ : Shocked material, $f$ : Shock wavefront, $g$ : Applicator, $h$ : Rectangular dielectric waveguide of $5 \mathrm{~m}$ in length, $i$ : Rectangular metallic waveguide, $j: 94 \mathrm{GHz}$ source, $k$ : Laser interferometer, $\vec{V}_{i m p}$ : Impact velocity, $\vec{V}_{1}$ : Shock wave velocity, $\vec{V}_{2}$ : Particle velocity

is incident upon this interface, the wave is partially reflected while the transmitted wave propagates behind the shock wavefront and is reflected by the aluminum coating deposited on the dielectric material under test. The dielectric applicator collects the reflected EM wave, which reaches the millimeter-wave source after a propagation along a dielectric waveguide. The mixing of the received signal with the transmitted millimeter-wave signal is then performed to derive the baseband signal. This signal is recorded with a sampling period of $0.4 \mathrm{~ns}$. As the PMMA is optically transparent, a laser interferometer with a collimating lens is placed on the same side as the dielectric applicator, and is used to measure the particle velocity for comparison purposes. The impact velocity $V_{\mathrm{imp}}$ is determined from recording the time of arrival of the projectile at different points in the gun barrel by using optical barriers. The setup allows addressing many impact velocities from $458 \mathrm{~m} \mathrm{~s}^{-1}$ to $658 \mathrm{~m} \mathrm{~s}^{-1}$ with applied pressure at the impact ranging from $8 \mathrm{kbar}$ to $12 \mathrm{kbar}$. Figures 1 is a photograph of the setup (the gun barrel is at the right of the picture). The Teflon applicator and optical lens are placed on one side of the dielectric material under test. The waveguide is embedded in an insulating foam. Fig. 2 shows a sketch of the setup. In this figure, the dashed line represents the shock front. The shock wave is generated at the impact of the impactor on the aluminum coating. Fig. 3 shows the typical baseband signal reflected by PMMA samples with $V_{\text {imp }}=520 \mathrm{~m} \mathrm{~s}^{-1}$. The impact time is $t_{i} \approx 218 \mu \mathrm{s}$. The shock reaches the rear of the sample at $t_{f} \approx 222.5 \mu$ s. Two oscillations at frequencies $f_{1}$ and $f_{2}$ $\left(<f_{1}\right)$ are clearly apparent in the measured baseband signal. These two frequencies are estimated here using the Fast Fourier Transform (FFT) and curve fitting based on the following profile:

$$
U(t)=A_{1} \sin \left(2 \pi f_{1} t+\varphi_{1}\right)+A_{1} \sin \left(2 \pi f_{2} t+\varphi_{2}\right)
$$

where $A_{1}$ and $A_{2}$ denotes (unknown) magnitudes, and $\varphi_{1}$ and $\varphi_{2}$ designates (unknown) phases in the reflected signal. The unique step of FFT is not sufficient here to determine these unknowns because of the very short measurement durations. Indeed, the transit time of the shock wave in the dielectric material under test is shorter than the period of the low frequency signal. Note that all experiments did not provide the two oscillations reported in Fig. 3. Only one observation is observed when the amplitude of the EM wave reflected by the shock wavefront is too weak to be detectable. We derived empirically that two oscillations are apparent only if the mass density contrast, and 
Figure 3: Amplitude of the signal reflected at $94 \mathrm{GHz}$ by a PMMA sample subjected to a shock loading with an impact velocity $V_{i m p}$ of $520 \mathrm{~m} \mathrm{~s}^{-1}$. Two oscillations at different frequencies are clearly apparent and allow deriving the particle and shock wave velocities.

therefore the permittivity contrast, between the unshocked and shocked PMMA material is at least $7.8 \%$, corresponding to an impact velocity of, at least $520 \mathrm{~m} \mathrm{~s}^{-1}$. Moreover, for impacts on an aluminum coating with a thickness of $200 \mathrm{~nm}$, an oscillation is observed before the impact. Assuming that this oscillation is related to the electromagnetic reflection on the impactor surface, the velocity of the projectile in the gun barrel can be estimated. As the aluminum thickness is smaller than the skin depth of $270 \mathrm{~nm}$ at $94 \mathrm{GHz}$, the incident EM wave is partially transmitted through the aluminum coating. With a thicker coating of $600 \mathrm{~nm}$, this oscillation is no more observable. Table 1 reports the impact velocities derived from the analysis of the baseband signal. An excellent agreement is obtained between the velocity estimated from optical barriers and one derived from the proposed millimeter-wave remote sensing technique (the difference does not exceed $3 \%$ for a measurement time of $10 \mu \mathrm{s})$. The larger uncertainty associated with the Doppler measurement is due to the very short time measurement duration. In addition, the particle velocity and the velocity of the shock wave can be derived. As a matter of fact, from the FFT applied to the reflected signal and curve fitting using eq. (1), the frequencies $f_{1}$ and $f_{2}$ of the two oscillations as well as the magnitude ratio $R_{a}=A_{2} / A_{1}$ can be computed. The results are reported in Table 2. As expected, due to skin depth effect, the shots $\mathrm{N}^{\circ} 4$ and $\mathrm{N}^{\circ} 5$ obtained from two different thicknesses of aluminum coating provide different magnitude ratios $R_{a}$. In the next section, the shock wavefront and the particle velocities are derived from these measurement results. 
Table 1: Impactor Velocity derived from Optical Barriers and from Millimeter-Wave Remote Sensing.

\begin{tabular}{|c|c|c|c|}
\hline $\begin{array}{l}\text { Shot's } \\
\text { rank }\end{array}$ & $\begin{array}{c}\text { Optical Barriers } \\
\text { Measurement } \\
\left(\mathrm{ms}^{-1}\right)\end{array}$ & $\begin{array}{l}\text { Doppler } \\
\text { Measureme } \\
\left(\mathrm{m} \mathrm{s}^{-1}\right)\end{array}$ & $\begin{array}{c}\text { Coating } \\
\text { Athickness } \\
\quad(\mu \mathrm{m})\end{array}$ \\
\hline 1 & $458 \pm 6$ & $\begin{array}{c}452 \pm \\
34.7\end{array}$ & 0.2 \\
\hline 2 & $520 \pm 8$ & $\begin{array}{c}528 \pm \\
13.3\end{array}$ & 0.2 \\
\hline 3 & $562 \pm 8$ & $\begin{array}{c}565 \pm \\
9.40\end{array}$ & 0.2 \\
\hline 4 & $663 \pm 9$ & $\begin{array}{c}667 \pm \\
13.3\end{array}$ & 0.2 \\
\hline 5 & $667 \pm 9$ & $\begin{array}{c}\text { not } \\
\text { measured }\end{array}$ & 0.6 \\
\hline
\end{tabular}

Table 2: The Computed Frequencies embedded in the Measured Baseband Signal and Amplitude Ratio $R_{a}$ for different Impact Conditions.

\begin{tabular}{ccccc}
\hline $\begin{array}{l}\text { Shot's } \\
\text { rank }\end{array}$ & $f_{1}(\mathrm{kHz})$ & $f_{2}(\mathrm{kHz})$ & $R_{a}$ & $\begin{array}{c}\text { Mass } \\
\text { Density } \\
\text { Gradient } \\
(\%)\end{array}$ \\
\hline 1 & $3280 \pm 5$ & $183 \pm 0.3$ & $29 \pm 1$ & 6.7 \\
2 & $3280 \pm 5$ & $183 \pm 0.3$ & $29 \pm 1$ & 7.8 \\
3 & $3340 \pm 14$ & $179 \pm 2$ & $27 \pm 1$ & 8.8 \\
4 & $3330 \pm 16.8$ & $221 \pm 2$ & $26 \pm 1$ & 10.0 \\
5 & $3290 \pm 17.8$ & $234 \pm 0.8$ & $47 \pm 1$ & 10.0 \\
\hline
\end{tabular}




\section{Derivation of the shock wavefront and the particle velocities from millimeter-wave remote sensing}

The millimeter-wave electric field reflected by the dielectric materials under shock loading combines mainly two contributions: the first contribution $\vec{E}_{\text {shock }}(z, t)$ is due to the reflection on the shock front moving interface and, assuming that multiple reflections inside the shocked sample are negligible, the second contribution $\vec{E}_{\text {particle }}(z, t)$ originates in the reflection from the impactor-dielectric material moving interface. The total electromagnetic reflected field can then be approximated as follows:

$$
\vec{E}_{r}(z, t)=\vec{E}_{\text {shock }}(z, t)+\vec{E}_{\text {particle }}(z, t)
$$

where the electric field reflected by the shock front interface is given by:

$$
\vec{E}_{\text {shock }}(z, t)=R_{1} \vec{E} e^{j 2 \pi f_{1} t+j \varphi_{1}}
$$

in which $R_{1}$ denotes the reflection coefficient on the shock wavefront moving interface, and $f_{1}$ and $\varphi_{1}$ are respectively the frequency and the phase shift of the field reflected by the moving shock wavefront. The second contribution to the total reflected field is as follows:

$$
\vec{E}_{\text {particle }}(z, t) \approx T_{1} R_{2} T_{2} \vec{E} e^{j 2 \pi f_{2} t+j \varphi_{2}}
$$

where $T_{1}$ and $T_{2}$ are the transmission coefficients through the moving shock wavefront, $R_{2}$ is the reflection coefficient on the impactor-dielectric material interface, and $f_{2}$ and $\varphi_{2}$ are respectively the frequency and the phase shift of the reflected electric field on the moving impactor-dielectric material interface. The detailed computation of Doppler frequency shifts and magnitude ratio $R_{a}$ allows the straightforward derivation of the shock wave velocity $V_{1}$, the particle velocity $V_{2}$ and the refractive index $n_{2}$ of the shocked material, as follows:

$$
\begin{aligned}
V_{1} & =\frac{c}{2 n_{1}} \frac{f_{1}}{f_{c}} \\
V_{2} & =V_{1}+\frac{c}{2 n_{2}} \frac{f_{2}-f_{1}}{f_{c}} \\
n_{2} & =\frac{-4 n_{1}\left(1-\frac{f_{1}-f_{2}}{f_{c}}\right)+\sqrt{\triangle}}{2 R_{a}}
\end{aligned}
$$

where $c$ is the light velocity in vacuum, $n_{1}(=1.64)$ is the known refractive index of the unshocked material, $n_{2}$ is the unknown refractive index of the shocked material, $f_{c}$ is the frequency of the incident millimeter-wave field and $\triangle$ is given as follows:

$$
\triangle=4 n_{1}^{2}\left(4\left[1-\frac{f_{1}-f_{2}}{f}\right]^{2}+R_{a}^{2}\right)
$$

The shock wave velocity $V_{1}$, the particle velocity $V_{2}$ and the refractive index $n_{2}$ of PMMA under shock loading can then be derived from eqs. (5)-(8) and from the measurement of $f_{1}, f_{2}$ and $R_{a}$. As it can be observed from Table 3 , the difference between the shock wave velocities obtained from the proposed millimiter-wave sensing technique and from 1D eulerian hydrocode simulation does not exceed $3 \%$. The 
Table 3: Shock Wave Velocity $V_{1}$ derived from Millimeter-Wave Remote Sensing and from 1D Hydrocode Simulation.

\begin{tabular}{ccc}
\hline Shot's rank & $V_{1}\left(\mathrm{~m} \mathrm{~s}^{-1}\right)$ & $\begin{array}{c}\text { 1D Hydrocode } \\
\text { Simulation results } \\
\left(\mathrm{m} \mathrm{s}^{-1}\right)\end{array}$ \\
\hline 2 & $3128 \pm 5$ & $3124 \pm 6$ \\
3 & $3185 \pm 13$ & $3155 \pm 6$ \\
4 & $3176 \pm 16$ & $3225 \pm 7$ \\
5 & $3138 \pm 17$ & $3228 \pm 7$ \\
\hline
\end{tabular}

Table 4: Comparison of the Measured Particle Velocity $V_{2}$ with Laser Interferometer Measurement.

\begin{tabular}{ccc}
\hline Shot's rank & $V_{2}\left(\mathrm{~m} \mathrm{~s}^{-1}\right)$ & $\begin{array}{c}\text { Laser Interferometer } \\
\text { measurement }\left(\mathrm{m} \mathrm{s}^{-1}\right)\end{array}$ \\
\hline 2 & $278 \pm 3$ & 263 \\
3 & $284 \pm 3$ & 281 \\
4 & $326 \pm 3$ & 326 \\
5 & $345 \pm 3$ & 334 \\
\hline
\end{tabular}

uncertainties are computed by propagating the error of the curve fitting for the remote sensing method. For the hydrocode computation, the uncertainty depends on the mesh size, which is here of $420 \mu \mathrm{m}$. Table 4 reports the estimation of particle velocities from the millimeter-wave remote sensing technique and from laser interferometer measurement. Again, the agreement is very good and the difference between these two velocity measurement techniques does not exceed $6 \%$. Moreover, from eq. (7), the refractive index $n_{2}$ of the PMMA subjected to an impact can be estimated and compared with the results derived from available theoretical models (see, e.g., [13]). The comparison between the available theoretical models and the obtained data is reported in Table 5. It can be observed that the Lorentz Lorenz model predicts results very close to our estimations with a maximum deviation of $1 \%$. This conclusion is consistent with the studies of the refractive index of PMMA under shock loading at visible frequencies reported in [14], where the authors conclude that the Lorentz Lorenz model is the most appropriate.

\section{Conclusion}

In this work, a millimeter-wave remote sensing technique is applied for deriving shock wave velocity, particle velocity and refractive index of shocked PMMA during plane mechanical impacts. This new technique brings many advantages as it does not require the prior knowledge of the permittivity of the shocked dielectric material under test. Experimental results on PMMA cylinders are reported. The shock wave and particle velocities are remotely derived and the results are found in very good agreement with 1D eulerian hydrocode simulations. Moreover, the derived particle velocity agrees very well with measurement provided by an optical interferometer. Finally, the refractive index of the shocked material is estimated and compared with available theoretical 
Table 5: Refractive Index $n_{2}$ of Shocked PMMA Samples derived from Millimeter-Wave Remote Sensing Technique and from Theoretical Models ${ }^{1}$.

\begin{tabular}{ccccc}
\hline Shot's rank & This work & \multicolumn{3}{c}{ GladstoneClausius } \\
& & Dale & $\begin{array}{c}\text { Lorentz } \\
\text { model } \\
\text { model }\end{array}$ & $\begin{array}{c}\text { Lorenz } \\
\text { model }\end{array}$ \\
\hline 2 & 3.01 & 2.97 & 3.09 & 3.01 \\
3 & 3.02 & 2.98 & 3.10 & 3.03 \\
4 & 3.03 & 2.99 & 3.14 & 3.06 \\
5 & 3.05 & 2.99 & 3.14 & 3.06 \\
\hline
\end{tabular}

${ }^{1}$ See [13] for the detailed description of the theoretical models considered here.

predictions. The results reported here demonstrate that millimeter-wave remote sensing technique can be advantageously applied to investigate physical phenomena at heart of shocked material samples. To investigate more complex cases such as the rarefaction waves, a model with a refractive index gradient should be developed.

\section{Acknowledgment}

The authors would like to thank Thomas Chazoule for his contribution on the theoretical part of the work and CEA engineers for their help during the measurement. This work was performed with financial support from CEA and the Occitanie region in France.

\section{References}

[1] M. A. Meyers, Dynamic Behavior of Materials. New York, NY: John Wiley and Sons, 1994

[2] J. Benterou, C. Bennett, G. Cole, D. Hare, C. May, and E. Udd, "Internal detonation velocity measurements inside high explosives," in SPIE Defense, Security and Sensing, Orlando, FL, USA, 2009.

[3] P. BRae, B. Glover, J. Gunderson, and L. Perry, "Free-field microwave interferometry for detonation front tracking and run-to-detonation measurements," in APS Shock Compression of Condensed Matter, Chicago, IL, USA, 2011.

[4] L. Barker and R. Hollenbach, "Shock wave studies of PMMA, fused silica and sapphire," Journal of Applied Physics, vol. 41, pp. 4208-4226, 1970.

[5] G. Collins, L. D. Silva, P. Celliers, D. Gold, M. Ford, R. Wallace, A. Weber, K. Budil, and R. Cauble, "Measurements of the equation of state of deuterium at the fluid insulator-metal transition," Science, vol. 281, pp. 1178-1181, 1998.

[6] R. Dick, "Shock wave compression of benzene, carbon disulfide, carbon tetrachloride, and liquid nitrogen," The Journal of Chemical Physics, vol. 52, no. 12 , pp. 6021-6032, 1970. 
[7] R. Gustavsen, S. Sheffield, and R. Alcon, "Response of inclined electromagnetic particle velocity gauges in shocked liquids," AIP Conference Proceedings, vol. 309, pp. 1703-1706, 1994.

[8] M. Cook, R. Doran, and G. Morris, "Measurement of detonation velocity by doppler effect at three-centimeter wavelength," Journal of Applied Physics, vol. 26, pp. 426-428, 1955.

[9] Microwave interferometer techniques for detonation study, 1985.

[10] G. G. Luther and B. J. Warthen, "Microwave interferometry to elucidate shock properties," AIP Conference Proceedings, vol. 309, no. 1, pp. 1755-1757, 1994.

[11] A. D. Krall, B. C. Glancy, and H. W. Sandusky, "Microwave interferometry of shock waves. i. unreacting porous media," Journal of Applied Physics, vol. 74, no. 10 , pp. 6322-6327, 1993.

[12] B. C. Glancy, H. W. Sandusky, and A. D. Krall, "Microwave interferometry of shock waves. ii. reacting porous media," Journal of Applied Physics, vol. 74, no. 10, pp. 6328-6334, 1993.

[13] R. S. Hawke, R. N. Keeler, and A. C. Mitchell, "Microwave dielectric constant of al2o3 at 375 kilobars," Applied Physics Letters, vol. 14, no. 7, pp. 229-231, 1969.

[14] D. J. Chapman, D. E. Eakins, D. M. Williamson, and W. Proud, "Index of refraction measurements and window corrections for pmma under shock compression," AIP Conference Proceedings, vol. 1426, no. 1, pp. 442-445, 2012. 